\title{
Singular control and optimal stopping of SPDEs, and backward SPDEs with reflection
}

\author{
Bernt Øksendal ${ }^{1,2}$
}

\author{
Agnès Sulem ${ }^{3}$,
}

30 September 2012
Tusheng Zhang ${ }^{4,1}$

\begin{abstract}
We consider general singular control problems for random fields given by a stochastic partial differential equation (SPDE). We show that under some conditions the optimal singular control can be identified with the solution of a coupled system of SPDE and a reflected backward SPDE (RBSPDE). As an illustration we apply the result to a singular optimal harvesting problem from a population whose density is modeled as a stochastic reaction-diffusion equation. Existence and uniqueness of solutions of RBSPDEs are established, which is of independent interest. We then establish a relation between RBSPDEs and optimal stopping of SPDEs, and apply the result to a risk minimizing stopping problem.
\end{abstract}

Keywords: Stochastic partial differential equations (SPDEs), singular control of SPDEs, maximum principles, comparison theorem for SPDEs, reflected SPDEs, optimal stopping of SPDEs.

$M S C(2010)$ : Primary 60H15 Secondary 93E20, 35R60.

\section{Introduction}

As a motivation for the problem studied here we consider a problem of optimal harvesting from a fish population in a lake D. Suppose the density $Y(t, x)$ of the population at time

\footnotetext{
${ }^{1}$ Center of Mathematics for Applications (CMA), Dept. of Mathematics, University of Oslo, P.O. Box 1053 Blindern, N-0316 Oslo, Norway, email: oksendal@math.uio.no. The research leading to these results has received funding from the European Research Council under the European Community's Seventh Framework Programme (FP7/2007-2013) / ERC grant agreement no [228087].

${ }^{2}$ Norwegian School of Economics and Business Administration, Helleveien 30, N-5045 Bergen, Norway.

${ }^{3}$ INRIA Paris-Rocquencourt, Domaine de Voluceau, Rocquencourt, BP 105, Le Chesnay Cedex, 78153, France, email: agnes.sulem@inria.fr

${ }^{4}$ School of Mathematics, University of Manchester, Oxford Road, Manchester M139PL, United Kingdom, email:Tusheng . zhang@manchester .ac.uk
} 
$t \in[0, T]$ and at the point $x \in D$ is given by a stochastic reaction-diffusion equation of the form

$$
\begin{aligned}
& d Y(t, x)=\left[\frac{1}{2} \Delta Y(t, x)+\alpha Y(t, x)\right] d t+\beta Y(t, x) d B(t)-\lambda_{0} Y(t, x) \xi(d t, x) ; \quad(t, x) \in(0, T) \times D \\
& Y(0, x)=y_{0}(x)>0 ; \quad x \in D \\
& Y(t, x)=0 ; \quad(t, x) \in(0, T) \times \partial D
\end{aligned}
$$

where $\mathrm{D}$ is a bounded domain in $\mathbb{R}^{d}$ and $y_{0}(x)$ is a given bounded deterministic function. Here $B(t)=B_{t}, t \geq 0$ is an $m$-dimensional Brownian motion on a filtered probability space $\left(\Omega, \mathcal{F}, \mathcal{F}_{t}, P\right), \alpha, \lambda_{0}>0$ are given constants, $\beta$ is a given vector and $\Delta:=\frac{1}{2} \sum_{i=1}^{d} \frac{\partial^{2}}{\partial x_{i}^{2}}$ is the Laplacian differential operator. We may regard $\xi(d t, x)$ as the harvesting effort rate and $\lambda_{0}>0$ as the relative harvesting efficiency coefficient. The performance coefficient is assumed to be

$$
J(\xi)=E\left[\int_{D} \int_{0}^{T}\left(h_{0}(t, x) Y(t, x)-c(t, x)\right) \xi(d t, x) d x+\int_{D} h_{0}(T, x) Y(T, x) d x\right],
$$

where $h_{0}(t, x)>0$ is the unit price of the fish and $c(t, x)$ is the unit cost of energy used in the harvesting and $T>0$ is a fixed terminal time. Thus $J(\xi)$ represents the expected total net income from the harvesting. The problem is to maximise $J(\xi)$ over all admissible harvesting strategies $\xi(t, x)$. We say that $\xi$ is admissible and write $\xi \in \mathcal{A}$ if $\xi(t, x)$ is $\mathcal{F}_{t}$ - adapted, nondecreasing in $t$ and $\xi(0, x)=0$ for each $x$. In this example we also require that the $t$-jumps of $\xi(t, x)$ are less than $\frac{1}{\lambda_{0}}$. This ensures that $Y(t, x)>0$ for all $(t, x) \in[0, T] \times(D \backslash \partial D)$.

The aim of this paper is to study singular control of stochastic partial differential equations (SPDE) driven by a multiplicative noise of finite dimension. In particular we want to establish a stochastic maximum principle and to study relations with some associated reflected backward SPDEs.

It is well-known that the stochastic maximum principle method for solving a stochastic control problem for SPDEs involves a backward SPDE for the adjoint processes $p(t, x), q(t, x)$ (see [ØPZ]). We will show that in the case of singular control problem for SPDE we arrive at a BSPDE with reflection for the adjoint processes.

Several papers are devoted to the study of backward SPDEs (without reflection) and maximum principle of SPDEs, see e.g. [B, HP1, HP2, HMY, GM]. In a finite dimensional setup, maximum principles for singular stochastic control problems have been studied in [AND, BM, BDM, BCDM] , and in the recent paper [ØS], where connections between singular stochastic control, reflected BSDEs and optimal information are also established. For the study of SPDEs with reflection, please see [DP1], [HP], [NP], [Z].

The paper is organized as follows: In Section 2, we study a class of singular control problems for SPDEs and prove a maximum principle for the solution of such problems. This maximum principle leads to an adjoint equation which is a reflected backward stochastic partial differential equation. Both the necessary and sufficient properties of the maximum principle are discussed and, similarly to the finite dimensional case, the sufficient condition is established under suitable concavity properties of the coefficients. 
As an illustration we apply the result to the singular optimal harvesting problem above. In Section 3, we study existence and uniqueness of backward stochastic partial differential equations (BSPDEs) with reflection. These results are of independent interest. In particular, a comparison theorem for BSPDEs is also proved in this secion. In Section 4, we establish connections between reflected BSPDEs and optimal stopping of SPDEs and in Section 5 we consider an application to a risk minimising stopping problem.

\section{Singular control of SPDEs}

Let $D$ be a bounded, regular domain in $\mathbb{R}^{d}$. Denote by $a(x)=\left(a_{i j}(x)\right)$ a matrix-valued function on $\mathbf{R}^{d}$ satisfying the uniform ellipticity condition:

$$
\frac{1}{c} I_{d} \leq a(x) \leq c I_{d} \quad \text { for some constant } c \in(0, \infty)
$$

Let $b(x)$ be a vector field on $D$ with $b \in L^{p}(D)$ for some $p>d$ and $q(x)$ a measurable real valued function on $D$ such that $q \in L^{p_{1}}(D)$ for some $p_{1}>\frac{d}{2}$. Introduce the following second order partial differential operator:

$$
A u=-\operatorname{div}(a(x) \nabla u(x))+b(x) \cdot \nabla u(x)+q(x) u(x) .
$$

Suppose the state equation is an SPDE of the form

$$
\begin{aligned}
d Y(t, x) & =\{A Y(t, x)+b(t, x, Y(t, x))\} d t+\sigma(t, x, Y(t, x)) d B(t) \\
& +\lambda(t, x, Y(t, x)) \xi(d t, x) ;(t, x) \in[0, T] \times D \\
Y(0, x) & =y_{0}(x) ; x \in D \\
Y(t, x) & =y_{1}(t, x) ;(t, x) \in(0, T) \times \partial D .
\end{aligned}
$$

Here $y_{0} \in K:=L^{2}(D)$ and $y_{1} \in L^{2}(D \times[0, T])$ are given functions. We assume that $b, \sigma$ and $\lambda$ are $C^{1}$ with respect to $y$. Let $V=W_{0}^{1,2}(D)$ be the Sobolev space of order one with zero boundary condition.

Then $Y$ is understood as a weak (variational) solution to $(2.1)$, in the sense that $y \in$ $C([0, T] ; K) \cap L^{2}([0, T] ; V)$ and for $\phi \in C_{0}^{\infty}(D)$,

$$
\begin{aligned}
<Y(t, \cdot), \phi>_{K}=< & y_{0}(\cdot), \phi>_{K}+\int_{0}^{t}<Y(s, \cdot), A^{*} \phi>d s+\int_{0}^{t}<b(s, \cdot, Y(s, \cdot)), \phi>_{K} d s \\
& +\int_{0}^{t}<\sigma(s, \cdot, Y(s, \cdot)), \phi>_{K} d B(s),
\end{aligned}
$$

where $A^{*}$ is the adjoint operator of $A,<,>$ denotes the dual pair between the space $V$ and its dual $V^{*}$. Under this framework the Itô formula can be applied to such SPDEs. See [P], $[\mathrm{PR}]$. 
The performance functional is given by

$$
\begin{aligned}
J(\xi) & =E\left[\int_{D} \int_{0}^{T} f(t, x, Y(t, x)) d t d x+\int_{D} g(x, Y(T, x)) d x\right. \\
& \left.+\int_{D} \int_{0}^{T} h(t, x, Y(t, x)) \xi(d t, x) d x\right],
\end{aligned}
$$

where $f(t, x, y), g(x, y)$ and $h(t, x, y)$ are bounded measurable functions which are differentiable in the argument $y$ and continuous w.r.t. $t$.

We want to maximise $J(\xi)$ over all $\xi \in \mathcal{A}$, where $\mathcal{A}$ is a given family of adapted processes $\xi(t, x)$, which are non-decreasing and left-continuous w.r.t. $t$ for all $x, \xi(0, x)=0$ and $\xi(T, x)<\infty$. We call $\mathcal{A}$ the set of admissible singular controls. Thus we want to find $\xi^{*} \in \mathcal{A}$ (called an optimal control) such that

$$
\sup _{\xi \in \mathcal{A}} J(\xi)=J\left(\xi^{*}\right)
$$

We study this problem by using an extension to SPDEs of the maximum principle in [ØS]: Define the Hamiltonian $H$ by

$$
\begin{gathered}
H(t, x, y, p, q)(d t, \xi(d t, x))=\{f(t, x, y)+b(t, x, y) p+\sigma(t, x, y) q\} d t \\
+\{\lambda(t, x, y) p+h(t, x, y)\} \xi(d t, x) .
\end{gathered}
$$

To this Hamiltonian we associate the following backward SPDE (BSPDE) in the unknown process $(p(t, x), q(t, x))$ :

$$
\begin{aligned}
d p(t, x) & =-\left\{A^{*} p(t, x) d t+\frac{\partial H}{\partial y}(t, x, Y(t, x), p(t, x), q(t, x))(d t, \xi(d t, x))\right\} \\
& +q(t, x) d B(t) ;(t, x) \in(0, T) \times D \\
p(T, x) & =\frac{\partial g}{\partial y}(x, Y(T, x)) ; x \in D \\
p(t, x) & =0 ;(t, x) \in(0, T) \times \partial D .
\end{aligned}
$$

Here $A^{*}$ denotes the adjoint of the operator $A$. We assume that a unique solution $p(t, x), q(t, x)$ of (2.6)-(2.8) exists for each $\xi \in \mathcal{A}$.

Theorem 2.1 (Sufficient maximum principle for singular control of SPDE) Let $\hat{\xi} \in$ $\mathcal{A}$ with corresponding solutions $\hat{Y}(t, x), \hat{p}(t, x), \hat{q}(t, x)$. Assume that

$y \rightarrow h(x, y)$ is concave,

$(y, \xi) \rightarrow H(t, x, y, \hat{p}(t, x), \hat{q}(t, x))(d t, \xi(d t, x))$ is concave,

$E\left[\int_{D}\left(\int_{0}^{T}\left\{\left(Y^{\xi}(t, x)-\hat{Y}(t, x)\right)^{2} \hat{q}^{2}(t, x)+\hat{p}^{2}(t, x)\left(\sigma\left(t, x, Y^{\xi}(t, x)\right)-\sigma(t, x, \hat{Y}(t, x))^{2}\right\} d t\right) d x\right]<\infty\right.$,

for all $\xi \in \mathcal{A}$. 
Moreover, assume that the following maximum condition holds:

$$
\hat{\xi}(d t, x) \in \underset{\xi \in \mathcal{A}}{\operatorname{argmax}} H(t, x, \hat{Y}(t, x), \hat{p}(t, x), \hat{q}(t, x))(d t, \xi(d t, x)),
$$

i.e.

$$
\begin{aligned}
& \{\lambda(t, x, \hat{Y}(t, x)) \hat{p}(t, x)+h(t, x, \hat{Y}(t, x))\} \xi(d t, x) \\
& \quad \leq\{\lambda(t, x, \hat{Y}(t, x)) \hat{p}(t, x)+h(t, x, \hat{Y}(t, x))\} \hat{\xi}(d t, x) \text { for all } \xi \in \mathcal{A} .
\end{aligned}
$$

Then $\hat{\xi}$ is an optimal singular control.

Proof of Theorem 2.1 Choose $\xi \in \mathcal{A}$ and put $Y=Y^{\xi}$. Then by (2.4) we can write

$$
J(\xi)-J(\hat{\xi})=I_{1}+I_{2}+I_{3},
$$

where

$$
\begin{gathered}
I_{1}=E\left[\int_{0}^{T} \int_{D}\{f(t, x, Y(t, x))-f(t, x, \hat{Y}(t, x))\} d x d t\right] \\
I_{2}=E\left[\int_{D}\{g(x, Y(T, x))-g(x, \hat{Y}(T, x))\} d x\right] \\
I_{3}=E\left[\int_{0}^{T} \int_{D}\{h(t, x, Y(t, x)) \xi(d t, x)-h(t, x, \hat{Y}(t, x)) \hat{\xi}(d t, x)\}\right] .
\end{gathered}
$$

By our definition of $H$ we have

$$
\begin{aligned}
I_{1}=E & {\left[\int_{0}^{T} \int_{D}\{H(t, x, Y(t, x), \hat{p}(t, x), \hat{q}(t, x))(d t, \hat{\xi}(d t, x))\right.} \\
& -H(t, x, \hat{Y}(t, x), \hat{p}(t, x), \hat{q}(t, x))(d t, \hat{\xi}(d t, x))\} \\
- & \int_{0}^{T} \int_{D}\{b(t, x, Y(t, x))-b(t, x, \hat{Y}(t, x)\} \hat{p}(t, x) d x d t \\
- & \int_{0}^{T} \int_{D}\{\sigma(t, x, Y(t, x))-\sigma(t, x, \hat{Y}(t, x))\} \hat{q}(t, x) d x d t \\
- & \int_{0}^{T} \int_{D} \hat{p}(t, x)\{\lambda(t, x, Y(t, x)) \xi(d t, x)-\lambda(t, x, \hat{Y}(t, x)) \hat{\xi}(d t, x)\} d x \\
- & \left.\int_{0}^{T} \int_{D}\{h(t, x, Y(t, x)) \xi(d t, x)-h(t, x, \hat{Y}(t, x)) \hat{\xi}(d t, x)\} d x\right] .
\end{aligned}
$$


By (2.11) and concavity of $g$ we have, with $\tilde{Y}=Y-\hat{Y}$,

$$
\begin{aligned}
I_{2} \leq & E\left[\int_{D} \frac{\partial g}{\partial y}(x, \hat{Y}(T, x))(Y(T, x)-\hat{Y}(T, x)) d x\right]=E\left[\int_{D} \hat{p}(T, x) \tilde{Y}(T, x) d x\right] \\
= & E\left[\int_{D} \int_{0}^{T} \tilde{Y}(t, x) d \hat{p}(t, x) d x+\int_{D} \int_{0}^{T} \hat{p}(t, x) d \tilde{Y}(t, x) d x\right. \\
& \left.+\int_{D} \int_{0}^{T}\{\sigma(t, x, Y(t, x))-\sigma(t, x, \hat{Y}(t, x))\} \hat{q}(t, x) d t d x\right] \\
= & E\left[\int_{D} \int_{0}^{T} \tilde{Y}(t, x)\left\{-A^{*} \hat{p}(t, x) d t-\frac{\partial H}{\partial y}(t, x, \hat{Y}, \hat{p}, \hat{q})(d t, \hat{\xi}(d t, x))\right\} d x\right. \\
& +\int_{D} \int_{0}^{T} \hat{p}(t, x)\{A \tilde{Y}(t, x)+b(t, x, Y(t, x))-b(t, x, \hat{Y}(t, x))\} d t d x \\
& +\int_{D} \int_{0}^{T} \hat{p}(t, x)\{\lambda(t, x, Y(t, x)) \xi(d t, x)-\lambda(t, x, \hat{Y}(t, x)) \hat{\xi}(d t, x)\} d x \\
& \left.+\int_{D} \int_{0}^{T}\{\sigma(t, x, Y(t, x))-\sigma(t, x, \hat{Y}(t, x))\} \hat{q}(t, x) d t d x\right] .
\end{aligned}
$$

The rigorous meaning of the expressions $\int_{D} \int_{0}^{T} \tilde{Y}(t, x) A^{*} \hat{p}(t, x) d t d x, \int_{D} \int_{0}^{T} \hat{p}(t, x) A \tilde{Y}(t, x) d t d x$ are

$$
\begin{gathered}
\int_{D} \int_{0}^{T} \tilde{Y}(t, x) A^{*} \hat{p}(t, x) d t d x=\int_{0}^{T}<\tilde{Y}(t, \cdot), A^{*} \hat{p}(t, \cdot)>d t \\
\int_{D} \int_{0}^{T} \hat{p}(t, x) A \tilde{Y}(t, x) d t d x=\int_{0}^{T}<\hat{p}(t, \cdot), A \tilde{Y}(t, \cdot)>d t
\end{gathered}
$$

here $<,>$ stands for the dual pair between the space $V=H_{0}^{1,2}(D)$ and its dual $V^{*}$.

In view of $<\tilde{Y}(t, \cdot), A^{*} \hat{p}(t, \cdot)>=<\hat{p}(t, \cdot), A \tilde{Y}(t, \cdot)>$, combining (2.14)-(2.19) and concavity of $H$, we have

$$
\begin{aligned}
J(\xi) & -J(\hat{\xi}) \leq E\left[\int_{D} \int_{0}^{T}\{H(t, x, Y(t, x), \hat{p}(t, x), \hat{q}(t, x))(d t, \xi(d t, x))\right. \\
& \left.\left.-H(t, x, \hat{Y}(t, x), \hat{p}(t, x), \hat{q}(t, x))(d t, \hat{\xi}(d t, x))-\tilde{Y}(t, x) \frac{\partial H}{\partial y}(t, x, \hat{Y}, \hat{p}, \hat{q})(d t, \hat{\xi}(d t, x))\right\} d x\right] \\
& \leq\left[\int_{D} \int_{0}^{T} \nabla_{\xi} H(t, x, \hat{Y}(t, x), \hat{p}(t, x), \hat{q}(t, x))(\xi(d t, x)-\hat{\xi}(d t, x)) d x\right] \\
& =E\left[\int_{D} \int_{0}^{T}\{\lambda(t, x, \hat{Y}(t, x)) \hat{p}(t, x)+h(t, x, \hat{Y}(t, x))\}(\xi(d t, x)-\hat{\xi}(d t, x)) d x\right] \\
& \leq 0 \text { by }(2.13) .
\end{aligned}
$$

This proves that $\hat{\xi}$ is optimal.

For $\xi \in \mathcal{A}$ we let $\mathcal{V}(\xi)$ denote the set of adapted processes $\zeta(t, x)$ of finite variation w.r.t. $t$ such that there exists $\delta=\delta(\xi)>0$ such that $\xi+y \zeta \in \mathcal{A}$ for all $y \in[0, \delta]$. 
Proceeding as in $[\varnothing \mathrm{S}]$ we prove the following useful result:

Lemma 2.2 The inequality (2.13) is equivalent to the following two variational inequalities:

$$
\begin{gathered}
\lambda(t, x, \hat{Y}(t, x)) \hat{p}(t, x)+h(t, x, \hat{Y}(t, x)) \leq 0 \text { for all } t, x \\
\{\lambda(t, x, \hat{Y}(t, x)) \hat{p}(t, x)+h(t, x, \hat{Y}(t, x))\} \hat{\xi}(d t, x)=0 \text { for all } t, x
\end{gathered}
$$

Proof. (i). Suppose (2.13) holds. Choosing $\xi=\hat{\xi}+y \zeta$ with $\zeta \in \mathcal{V}(\hat{\xi})$ and $y \in(0, \delta(\hat{\xi}))$ we deduce that

$$
\{\lambda(s, x, \hat{Y}(s, x)) \hat{p}(s, x)+h(s, x, \hat{Y}(s, x))\} \zeta(d s, x) \leq 0 ;(s, x) \in(0, T) \times D
$$

for all $\zeta \in \mathcal{V}(\hat{\xi})$.

In particular, this holds if we fix $t \in(0, T)$ and put

$$
\zeta(d s, x)=a(\omega) \delta_{t}(d s) \phi(x) ;(s, x, \omega) \in(0, T) \times D \times \Omega,
$$

where $a(\omega) \geq 0$ is $\mathcal{F}_{t^{-}}$measurable and bounded, $\phi(x) \geq 0$ is bounded, deterministic and $\delta_{t}(d s)$ denotes the Dirac measure at $t$. Note that $\zeta \in \mathcal{V}(\xi)$. Then we get

$$
\lambda(t, x, \hat{Y}(t, x)) \hat{p}(t, x)+h(t, x, \hat{Y}(t, x)) \leq 0 \text { for all } \quad t, x
$$

which is $(2.20)$.

On the other hand, clearly $\zeta(d t, x):=\hat{\xi}(d t, x) \in \mathcal{V}(\hat{\xi})$ and this choice of $\zeta$ in (2.22) gives

$$
\{\lambda(t, x, \hat{Y}(t, x)) \hat{p}(t, x)+h(t, x, \hat{Y}(t, x))\} \hat{\xi}(d t, x) \leq 0 ;(t, x) \in(0, T) \times D
$$

Similarly, we can choose $\zeta(d t, x)=-\hat{\xi}(d t, x) \in \mathcal{V}(\hat{\xi})$ and this gives

$$
\{\lambda(t, x, \hat{Y}(t, x)) \hat{p}(t, x)+h(t, x, \hat{Y}(t, x))\} \hat{\xi}(d t, x) \geq 0 ;(t, x) \in(0, T) \times D
$$

Combining (2.24) and (2.25) we get

$$
\{\lambda(t, x, \hat{Y}(t, x)) \hat{p}(t, x)+h(t, x, \hat{Y}(t, x))\} \hat{\xi}(d t, x)=0
$$

which is (2.21). Together with (2.23) this proves (i).

(ii). Conversely, suppose (2.20) and (2.21) hold. Since $\xi(d t, x) \geq 0$ for all $\xi \in \mathcal{A}$ we see that (2.13) follows.

We may formulate what we have proved as follows:

Theorem 2.3 (Sufficient maximum principle II) Suppose the conditions of Theorem 2.1 hold. Suppose $\xi \in \mathcal{A}$, and that $\xi$ together with its corresponding processes $Y^{\xi}(t, x), p^{\xi}(t, x), q^{\xi}(t, x)$ 
solve the coupled system consisting of the SPDE (2.1)-(2.2) together with the reflected backward SPDE (RBSPDE) given by

$$
\begin{aligned}
& d p^{\xi}(t, x)=-\left\{A^{*} p^{\xi}(t, x)+\frac{\partial f}{\partial y}\left(t, x, Y^{\xi}(t, x)\right)+\frac{\partial b}{\partial y}\left(t, x, Y^{\xi}(t, x)\right) p^{\xi}(t, x)+\frac{\partial \sigma}{\partial y}\left(t, x, Y^{\xi}(t, x)\right) q^{\xi}(t, x)\right\} d t \\
& -\left\{\frac{\partial \lambda}{\partial y}\left(t, x, Y^{\xi}(t, x)\right) p^{\xi}(t, x)+\frac{\partial h}{\partial y}\left(t, x, Y^{\xi}(t, x)\right)\right\} \xi(d t, x)+q(t, x) d B(t) ;(t, x) \in[0, T] \times D \\
& \lambda\left(t, x, Y^{\xi}(t, x)\right) p^{\xi}(t, x)+h\left(t, x, Y^{\xi}(t, x)\right) \leq 0 ; \text { for all } t, x, \text { a.s. } \\
& \left\{\lambda\left(t, x, Y^{\xi}(t, x)\right) p^{\xi}(t, x)+h\left(t, x, Y^{\xi}(t, x)\right)\right\} \xi(d t, x)=0 ; \text { for all } t, x, \text { a.s. } \\
& p(T, x)=\frac{\partial g}{\partial y}\left(x, Y^{\xi}(T, x)\right) ; x \in D \\
& p(t, x)=0 ;(t, x) \in(0, T) \times \partial D .
\end{aligned}
$$

Then $\xi$ maximises the performance functional $J(\xi)$.

It is also of interest to have a maximum principle of "necessary type". To this end, we first prove some auxiliary results.

Lemma 2.4 Let $\xi(d t, x) \in \mathcal{A}$ and choose $\zeta(d t, x) \in \mathcal{V}(\xi)$. Define the derivative process

$$
\mathcal{Y}(t, x)=\lim _{y \rightarrow 0^{+}} \frac{1}{y}\left(Y^{\xi+y \zeta}(t, x)-Y^{\xi}(t, x)\right) .
$$

Then $\mathcal{Y}$ satisfies the SPDE

$$
\begin{aligned}
& d \mathcal{Y}(t, x)=A \mathcal{Y}(t, x) d t+\mathcal{Y}(t, x)\left[\frac{\partial b}{\partial y}(t, x, Y(t, x)) d t\right. \\
& \left.\quad+\frac{\partial \sigma}{\partial y}(t, x, Y(t, x)) d B(t)+\frac{\partial \lambda}{\partial y}(t, x, Y(t, x)) \xi(d t, x)\right] \\
& \quad+\lambda(t, x, Y(t, x)) \zeta(d t, x) ; \quad(t, x) \in[0, T] \times D \\
& \mathcal{Y}(t, x)=0 ; \quad(t, x) \in(0, T) \times \partial D \\
& \mathcal{Y}(0, x)=0 ; \quad x \in D
\end{aligned}
$$

Proof. This follows from the equation (2.1)-(2.2) for $Y(t, x)$. We omit the details.

Lemma 2.5 Let $\xi(d t, x) \in \mathcal{A}$ and $\zeta(d t, x) \in \mathcal{V}(\xi)$. Put $\eta=\xi+y \zeta ; y \in[0, \delta(\xi)]$. Assume that

$$
\begin{aligned}
& E\left[\int _ { D } \left(\int _ { 0 } ^ { T } \left\{\left(Y^{\eta}(t, x)-Y^{\xi}(t, x)\right)^{2} q^{2}(t, x)\right.\right.\right. \\
& \left.\left.\left.\quad+p^{2}(t, x)\left(\sigma\left(t, x, Y^{\eta}(t, x)\right)-\sigma\left(t, x, Y^{\xi}(t, x)\right)\right)^{2}\right\} d t\right) d x\right]<\infty \text { for all } y \in[0, \delta(\xi)]
\end{aligned}
$$


where $(p(t, x), q(t, x))$ is the solution of (2.5)-(2.7) corresponding to $Y^{\xi}(t, x)$. Then

$$
\begin{aligned}
\lim _{y \rightarrow 0^{+}} \frac{1}{y}(J(\xi+y \zeta)-J(\xi)) \\
\quad=E\left[\int_{D}\left(\int_{0}^{T}\{\lambda(t, x, Y(t, x)) p(t, x)+h(t, x, Y(t, x))\} \zeta(d t, x)\right) d x\right] .
\end{aligned}
$$

Proof. By (2.3) and (2.26), we have

$$
\begin{aligned}
\lim _{y \rightarrow 0^{+}} \frac{1}{y}( & J(\xi+y \zeta)-J(\xi)) \\
& =E\left[\int_{D}\left\{\int_{0}^{T} \frac{\partial f}{\partial y}(t, x, Y(t, x)) \mathcal{Y}(t, x) d t+\frac{\partial g}{\partial y}(x, Y(T, x)) \mathcal{Y}(T, x)\right\} d x\right. \\
& \left.+\int_{D} \int_{0}^{T} \frac{\partial h}{\partial y}(t, x, Y(t, x)) \mathcal{Y}(t, x) \xi(d t, x) d x+\int_{D} \int_{0}^{T} h(t, x, Y(t, x)) \zeta(d t, x) d x\right]
\end{aligned}
$$

By (2.4) and (2.27) we obtain

$$
\begin{array}{r}
E\left[\int_{D} \int_{0}^{T} \frac{\partial f}{\partial y}(t, x, Y(t, x)) \mathcal{Y}(t, x) d t d x\right] \\
=E\left[\int _ { D } \left(\int _ { 0 } ^ { T } \mathcal { Y } ( t , x ) \left\{\frac{\partial H}{\partial y}(d t, \xi(d t, x))-p(t, x) \frac{\partial b}{\partial y}(t, x) d t\right.\right.\right. \\
\left.\left.-q(t, x) \frac{\partial \sigma}{\partial y}(t, x) d t-\left(p(t, x) \frac{\partial \lambda}{\partial y}(t, x)+\frac{\partial h}{\partial y}(t, x)\right) \xi(d t, x)\right\}\right) d x,
\end{array}
$$

where we have used the abbreviated notation

$$
\frac{\partial H}{\partial y}(d t, \xi(d t, x))=\frac{\partial H}{\partial y}(t, x, Y(t, x), p(t, x), q(t, x))(d t, \xi(d t, x))
$$

etc. 
By the Itô formula and (2.5), (2.28) we see that

$$
\begin{aligned}
& E\left[\int_{D} \frac{\partial g}{\partial y}(x) \mathcal{Y}(T, x) d x\right] \\
& =E\left[\int_{D} p(T, x) \mathcal{Y}(T, x) d x\right] \\
& =E\left[\int_{D}\left(\int_{0}^{T}\{p(t, x) d \mathcal{Y}(t, x)+\mathcal{Y}(t, x) d p(t, x)\}+[p(\cdot, x), \mathcal{Y}(\cdot, x)](T)\right) d x\right] \\
& =E\left[\int _ { D } \left(\int _ { 0 } ^ { T } \left[p ( t , x ) \left\{A \mathcal{Y}(t, x) d t+\mathcal{Y}(t, x) \frac{\partial b}{\partial y}(t, x) d t\right.\right.\right.\right. \\
& \left.\quad+\mathcal{Y}(t, x) \frac{\partial \lambda}{\partial y}(t, x) \xi(d t, x)+\lambda(t, x) \zeta(d t, x)\right\} \\
& +\mathcal{Y}(t, x)\left\{-A^{*} p(t, x) d t-\frac{\partial H}{\partial y}(d t, \xi(d t, x))\right\} \\
& \left.\left.\left.+\mathcal{Y}(t, x) \frac{\partial \sigma}{\partial y}(t, x) q(t, x)\right] d t\right) d x\right]
\end{aligned}
$$

where $[p(\cdot, x), \mathcal{Y}(\cdot, x)](t)$ denotes the covariation process of $p(\cdot, x)$ and $\mathcal{Y}(\cdot, x)$.

Since $p(t, x)=\mathcal{Y}(t, x)=0$ for $x \in \partial D$, we deduce that

$$
\int_{D} p(t, x) A \mathcal{Y}(t, x) d x=\int_{D} A^{*} p(t, x) \mathcal{Y}(t, x) d x .
$$

Therefore, substituting (2.31) and (2.32) into (2.30), we get

$$
\begin{aligned}
\lim _{y \rightarrow 0^{+}} \frac{1}{y}(J(\xi+y \zeta)-J(\xi)) \\
\quad=E\left[\int_{D}\left(\int_{0}^{T}\{\lambda(t, x) p(t, x)+h(t, s)\} \zeta(d t, x)\right) d x\right] .
\end{aligned}
$$

We can now state our necessary maximum principle:

Theorem 2.6 [Necessary maximum principle]

(i) Suppose $\xi^{*} \in \mathcal{A}$ is optimal, i.e. $\max _{\xi \in \mathcal{A}} J(\xi)=J\left(\xi^{*}\right)$. Let $Y^{*},\left(p^{*}, q^{*}\right)$ be the corresponding solution of (2.1)-(2.2) and (2.5)-(2.7), respectively, and assume that (2.28) holds with $\xi=\xi^{*}$. Then

$$
\lambda\left(t, x, Y^{*}(t, x)\right) p^{*}(t, x)+h\left(t, x, Y^{*}(t, x)\right) \leq 0 \quad \text { for all } t, x \in[0, T] \times D \text {, a.s. }
$$

and

$$
\left\{\lambda\left(t, x, Y^{*}(t, x)\right) p^{*}(t, x)+h\left(t, x, Y^{*}(t, x)\right)\right\} \xi^{*}(d t, x)=0 \quad \text { for all } t, x \in[0, T] \times D, \text { a.s. }
$$


(ii) Conversely, suppose that there exists $\hat{\xi} \in \mathcal{A}$ such that the corresponding solutions $\hat{Y}(t, x),(\hat{p}(t, x), \hat{q}(t, x))$ of (2.1)-(2.2) and (2.5)-(2.7), respectively, satisfy

$$
\lambda(t, x, \hat{Y}(t, x)) \hat{p}(t, x)+h(t, x, \hat{Y}(t, x)) \leq 0 \quad \text { for all } t, x \in[0, T] \times D, \text { a.s. }
$$

and

$$
\{\lambda(t, x, \hat{Y}(t, x)) \hat{p}(t, x)+h(t, x, \hat{Y}(t, x))\} \hat{\xi}(d t, x)=0 \quad \text { for all } t, x \in[0, T] \times D, \text { a.s. }
$$

Then $\hat{\xi}$ is a directional sub-critical point for $J(\cdot)$, in the sense that

$$
\lim _{y \rightarrow 0^{+}} \frac{1}{y}(J(\hat{\xi}+y \zeta)-J(\hat{\xi})) \leq 0 \quad \text { for all } \zeta \in \mathcal{V}(\hat{\xi})
$$

Proof. This is proved in a similar way as in Theorem 2.4 in [ØS]. For completeness we give the details:

(i) If $\xi \in \mathcal{A}$ is optimal, we get by Lemma 2.5

$$
\begin{aligned}
0 & \geq \lim _{y \rightarrow 0^{+}} \frac{1}{y}(J(\xi+y \zeta)-J(\xi)) \\
& =E\left[\int_{D} \int_{0}^{T}\{\lambda(t, x) p(t, x)+h(t, x)\} \zeta(d t, x) d x\right], \quad \text { for all } \zeta \in \mathcal{V}(\xi) .
\end{aligned}
$$

In particular, this holds if we choose $\zeta$ such that

$$
\zeta(d s, x)=a(\omega) \delta_{t}(s) \phi(x)
$$

for some fixed $t \in[0, T]$ and some bounded $\mathcal{F}_{t}$-measurable random variable $a(\omega) \geq 0$ and some bounded, deterministic $\phi(x) \geq 0$, where $\delta_{t}(s)$ is Dirac measure at $t$. Then (2.39) gets the form

$$
E\left[\int_{D}\{\lambda(t, x) p(t, x)+h(t, x)\} a(\omega) \phi(x) d x\right] \leq 0 .
$$

Since this holds for all such $a(\omega), \phi(x)$ we deduce that

$$
\lambda(t, x) p(t, x)+h(t, x) \leq 0 \quad \text { for all } t, x, a . s .
$$

Next, if we choose $\zeta(d t, x)=\xi(d t, x) \in \mathcal{V}(\xi)$, we get from $(2.39)$

$$
E\left[\int_{D} \int_{0}^{T}\{\lambda(t, x) p(t, x)+h(t, x)\} \xi(d t, x) d x\right] \leq 0 .
$$

On the other hand, we can also choose $\zeta(d t, x)=-\xi(d t, x) \in \mathcal{V}(\xi)$, and this gives

$$
E\left[\int_{D} \int_{0}^{T}\{\lambda(t, x) p(t, x)+h(t, x)\} \xi(d t, x) d x\right] \geq 0 .
$$


Combining (2.42) and (2.43) we get

$$
E\left[\int_{D} \int_{0}^{T}\{\lambda(t, x) p(t, x)+h(t, x)\} \xi(d t, x) d x\right]=0 .
$$

Combining (2.41) and (2.44) we see that

$$
\{\lambda(t, x) p(t, x)+h(t, x)\} \xi(d t, x)=0 \quad \text { for all } t, x, \text { a.s. }
$$

as claimed. This proves (i).

(ii) Conversely, suppose $\hat{\xi} \in \mathcal{A}$ is as in (ii). Then (2.38) follows from Lemma 2.5.

\subsection{Application to Optimal Harvesting}

We now return to the problem of optimal harvesting from a fish population in a lake D stated in the Introduction. Thus we suppose the density $Y(t, x)$ of the population at time $t \in[0, T]$ and at the point $x \in D$ is given by the stochastic reaction-diffusion equation (1.1), and the performance criterion is assumed to be as in (1.2). In this case the Hamiltonian is

$$
\begin{aligned}
& H(t, x, y, p, q)(d t, \xi(d t, x)) \\
& =(\alpha y p+\beta y q) d t+\left[-\lambda_{0} y p+h_{0}(t, x) y-c(t, x)\right] \xi(d t, x)
\end{aligned}
$$

and the adjoint equation is

$$
\begin{aligned}
& d p(t, x)=-\left[\frac{1}{2} \Delta p(t, x)+\alpha p(t, x)+\beta q(t, x)\right] d t \\
& +\left[\lambda_{0} p(t, x)-h_{0}(t, x)\right] \xi(d t, x)+q(t, x) d B(t, x) ; \quad(t, x) \in(0, T) \times D, \\
& p(T, x)=h_{0}(T, x) ; \quad x \in D \\
& p(t, x)=0 ; \quad(t, x) \in(0, T) \times \partial D .
\end{aligned}
$$

The variational inequalities for an optimal control $\xi(d t, x)$ are:

$$
\begin{aligned}
& -\lambda_{0} Y(t, x) p(t, x)+h_{0}(t, x) Y(t, x)-c(t, x) \leq 0 ; \quad(t, x) \in[0, T] \times D, \\
& {\left[-\lambda_{0} Y(t, x) p(t, x)+h_{0}(t, x) Y(t, x)-c(t, x)\right] \xi(d t, x)=0 ; \quad(t, x) \in[0, T] \times D}
\end{aligned}
$$

Since $Y(t, x)>0$ (by assumption on $\xi$ ), we can rewrite the variational inequalities above as follows:

$$
\begin{aligned}
& p(t, x) \geq \frac{h_{0}(t, x)}{\lambda_{0}}-\frac{c(t, x)}{\lambda_{0} Y(t, x)} ; \quad(t, x) \in[0, T] \times(D \backslash \partial D) \\
& {\left[p(t, x)-\frac{h_{0}(t, x)}{\lambda_{0}}-\frac{c(t, x)}{\lambda_{0} Y(t, x)}\right] \xi(d t, x)=0 ; \quad(t, x) \in[0, T] \times(D \backslash \partial D) .}
\end{aligned}
$$

We summerise the above in the following: 
Theorem 2.7 (a) Suppose $\xi(d t, x) \in \mathcal{A}$ is an optimal singular control for the harvesting problem

$$
\sup _{\xi \in \mathcal{A}} E\left[\int_{D} \int_{0}^{T}\left(h_{0}(t, x) Y(t, x)-c(t, x)\right) \xi(d t, x) d x+\int_{D} h_{0}(T, x) Y(t, x) d x\right]
$$

where $Y(t, x)$ is given by the SPDE (1.1). Then $\xi(d t, x)$ solves the reflected BSPDE (2.47), $(2.50)$.

(b) Conversely, suppose $\xi(d t, x)$ is a solution of the reflected BSPDE (2.47), (2.50). Then $\xi(d t, x)$ is a directional sub-critical point optimal control for the performance $J(\cdot)$ given by $(1.2)$.

Heuristically we can interpret the optimal harvesting strategy as follows:

- As long as $p(t, x)>\frac{h_{0}(t, x)}{\lambda_{0}}-\frac{c(t, x)}{\lambda_{0} Y(t, x)}$, we do nothing

- If $p(t, x)=\frac{h_{0}(t, x)}{\lambda_{0}}-\frac{c(t, x)}{\lambda_{0} Y(t, x)}$, we harvest immediately from $\mathrm{Y}(\mathrm{t}, \mathrm{x})$ at a rate $\xi(d t, x)$ which is exactly enough to prevent $p(t, x)$ from dropping below $\frac{h_{0}(t, x)}{\lambda_{0}}-\frac{c(t, x)}{\lambda_{0} Y(t, x)}$ in the next moment

- If $p(t, x)<\frac{h_{0}(t, x)}{\lambda_{0}}-\frac{c(t, x)}{\lambda_{0} Y(t, x)}$, we harvest immediately what is necessary to bring $p(t, x)$ up to the level of $\frac{h_{0}(t, x)}{\lambda_{0}}-\frac{c(t, x)}{\lambda_{0} Y(t, x)}$.

Note that an immediate harvesting of an amount $\Delta \xi>0$ from $Y(t, x)$ produces an immediate increase in the difference $\Delta W$ of the process

$$
W(t, x):=p(t, x)-\frac{h_{0}(t, x)}{\lambda_{0}}+\frac{c(t, x)}{\lambda_{0} Y(t, x)} .
$$

\section{Existence and uniqueness results of reflected back- ward SPDEs}

In this section we prove the main existence and uniqueness result for reflected backward stochastic partial differential equations. For notational simplicity, we choose the operator $A$ to be the Laplacian operator $\Delta$. However, our methods work equally well for general second order differential operators like

$$
A=\frac{1}{2} \sum_{i, j=1}^{d} \frac{\partial}{\partial x_{i}}\left(a_{i j}(x) \frac{\partial}{\partial x_{j}}\right)
$$

where $a=\left(a_{i j}(x)\right): D \rightarrow \mathbb{R}^{d \times d}(d>2)$ is a measurable, symmetric matrix-valued function which satisfies the uniform ellipticity condition

$$
\lambda|z|^{2} \leq \sum_{i, j=1}^{d} a_{i j}(x) z_{i} z_{j} \leq \Lambda|z|^{2}, \forall z \in \mathbb{R}^{d} \text { and } x \in D
$$


for some constants $\lambda, \Lambda>0$

First we will establish a comparison theorem for BSPDEs, which is of independent interest. Consider two backward SPDEs:

$$
\begin{aligned}
d u_{1}(t, x) & =-\frac{1}{2} \Delta u_{1}(t, x) d t-b_{1}\left(t, u_{1}(t, x), Z_{1}(t, x)\right) d t+Z_{1}(t, x) d B_{t}, t \in(0, T) \\
u_{1}(T, x) & =\phi_{1}(x) \quad \text { a.s. } \\
d u_{2}(t, x) & =-\frac{1}{2} \Delta u_{2}(t, x) d t-b_{2}\left(t, u_{2}(t, x), Z_{2}(t, x)\right) d t+Z_{2}(t, x) d B_{t}, t \in(0, T) \\
u_{2}(T, x) & =\phi_{2}(x) \quad \text { a.s. }
\end{aligned}
$$

From now on, if $u(t, x)$ is a function of $(t, x)$, we write $u(t)$ for the function $u(t, \cdot)$.

The following result is a comparison theorem for backward stochastic partial differential equations.

Theorem 3.1 (Comparison theorem for BSPDEs) Suppose $\phi_{1}(x) \leq \phi_{2}(x)$ and $b_{1}(t, u, z) \leq$ $b_{2}(t, u, z)$. Then we have $u_{1}(t, x) \leq u_{2}(t, x), x \in D$, a.e. for every $t \in[0, T]$.

Proof. For $n \geq 1$, define functions $\psi_{n}(z), f_{n}(x)$ as follows (see [DP1]).

$$
\begin{gathered}
\psi_{n}(z)= \begin{cases}0 & \text { if } z \leq 0, \\
2 n z & \text { if } 0 \leq z \leq \frac{1}{n} \\
2 & \text { if } z>\frac{1}{n}\end{cases} \\
f_{n}(x)= \begin{cases}0 & \text { if } x \leq 0, \\
\int_{0}^{x} d y \int_{0}^{y} \psi_{n}(z) d z & \text { if } x>0 .\end{cases}
\end{gathered}
$$

We have

$$
f_{n}^{\prime}(x)= \begin{cases}0 & \text { if } x \leq 0, \\ n x^{2} & \text { if } x \leq \frac{1}{n}, \\ 2 x-\frac{1}{n} & \text { if } x>\frac{1}{n} .\end{cases}
$$

Also $f_{n}(x) \uparrow\left(x^{+}\right)^{2}$ as $n \rightarrow \infty$. For $h \in K:=L^{2}(D)$, set

$$
F_{n}(h)=\int_{D} f_{n}(h(x)) d x .
$$

$F_{n}$ has the following derivatives for $h_{1}, h_{2} \in K$,

$$
\begin{gathered}
F_{n}^{\prime}(h)\left(h_{1}\right)=\int_{D} f_{n}^{\prime}(h(x)) h_{1}(x) d x, \\
F_{n}^{\prime \prime}(h)\left(h_{1}, h_{2}\right)=\int_{D} f_{n}^{\prime \prime}(h(x)) h_{1}(x) h_{2}(x) d x .
\end{gathered}
$$


Applying Ito's formula we get

$$
\begin{aligned}
& F_{n}\left(u_{1}(t)-u_{2}(t)\right) \\
= & F_{n}\left(\phi_{1}-\phi_{2}\right)+\int_{t}^{T} F_{n}^{\prime}\left(u_{1}(s)-u_{2}(s)\right)\left(\Delta\left(u_{1}(s)-u_{2}(s)\right)\right) d s \\
& +\int_{t}^{T} F_{n}^{\prime}\left(u_{1}(s)-u_{2}(s)\right)\left(b_{1}\left(s, u_{1}(s), Z_{1}(s)\right)-b_{2}\left(s, u_{2}(s), Z_{2}(s)\right)\right) d s \\
& -\int_{t}^{T} F_{n}^{\prime}\left(u_{1}(s)-u_{2}(s)\right)\left(Z_{1}(s)-Z_{2}(s)\right) d B_{s} \\
& -\frac{1}{2} \int_{t}^{T} F_{n}^{\prime \prime}\left(u_{1}(s)-u_{2}(s)\right)\left(Z_{1}(s)-Z_{2}(s), Z_{1}(s)-Z_{2}(s)\right) d s \\
=: & I_{n}^{1}+I_{n}^{2}+I_{n}^{3}+I_{n}^{4}+I_{n}^{5},
\end{aligned}
$$

where,

$$
\begin{gathered}
I_{n}^{2}=\int_{t}^{T} F_{n}^{\prime}\left(u_{1}(s)-u_{2}(s)\right)\left(\Delta\left(u_{1}(s)-u_{2}(s)\right)\right) d s \\
=\int_{t}^{T} \int_{D} f_{n}^{\prime}\left(u_{1}(s, x)-u_{2}(s, x)\right)\left(\Delta\left(u_{1}(s, x)-u_{2}(s, x)\right)\right) d x d s \\
=-\int_{t}^{T} f_{n}^{\prime \prime}\left(u_{1}(s, x)-u_{2}(s, x)\right) \mid \nabla\left(u_{1}(s, x)-\left.u_{2}(s, x)\right|^{2} d x d s \leq 0,\right. \\
I_{n}^{5}=-n \int_{t}^{T} \int_{D} \chi_{\left\{0 \leq u_{1}(s, x)-u_{2}(s, x) \leq \frac{1}{n}\right\}}\left(u_{1}(s, x)-u_{2}(s, x)\right)\left|Z_{1}(s, x)-Z_{2}(s, x)\right|^{2} d x d s \\
-\int_{t}^{T} \int_{D} \chi_{\left\{u_{1}(s, x)-u_{2}(s, x)>\frac{1}{n}\right\}}\left|Z_{1}(s, x)-Z_{2}(s, x)\right|^{2} d x d s .
\end{gathered}
$$

For $I_{n}^{3}$, we have

$$
\begin{aligned}
I_{n}^{3}= & \int_{t}^{T} \int_{D} f_{n}^{\prime}\left(u_{1}(s, x)-u_{2}(s, x)\right)\left(b_{1}\left(s, u_{1}(s, x), Z_{1}(s, x)\right)-b_{2}\left(s, u_{2}(s, x), Z_{2}(s, x)\right)\right) d x d s \\
= & \int_{t}^{T} \int_{D} f_{n}^{\prime}\left(u_{1}(s, x)-u_{2}(s, x)\right)\left(b_{1}\left(s, u_{1}(s, x), Z_{1}(s, x)\right)-b_{2}\left(s, u_{1}(s, x), Z_{1}(s, x)\right)\right) d x d s \\
& +\int_{t}^{T} \int_{D} f_{n}^{\prime}\left(u_{1}(s, x)-u_{2}(s, x)\right)\left(b_{2}\left(s, u_{1}(s, x), Z_{1}(s, x)\right)-b_{2}\left(s, u_{2}(s, x), Z_{1}(s, x)\right)\right) d x d s \\
& +\int_{t}^{T} \int_{D} f_{n}^{\prime}\left(u_{1}(s, x)-u_{2}(s, x)\right)\left(b_{2}\left(s, u_{2}(s, x), Z_{1}(s, x)\right)-b_{2}\left(s, u_{2}(s, x), Z_{2}(s, x)\right)\right) d x d s \\
\leq & \int_{t}^{T} \int_{D} f_{n}^{\prime}\left(u_{1}(s, x)-u_{2}(s, x)\right)\left(b_{2}\left(s, u_{2}(s, x), Z_{1}(s, x)\right)-b_{2}\left(s, u_{2}(s, x), Z_{2}(s, x)\right)\right) d x d s \\
& +C \int_{t}^{T} \int_{D}\left(\left(u_{1}(s, x)-u_{2}(s, x)^{+}\right)^{2} d x d s:=I_{n, 1}^{3}+I_{n, 2}^{3},\right.
\end{aligned}
$$


where the Lipschiz condition of $b$ and the assumption $b_{1} \leq b_{2}$ have been used. $I_{n, 1}^{3}$ can be estimated as follows:

$$
\begin{aligned}
I_{n, 1}^{3} \leq & C \int_{t}^{T} \int_{D} f_{n}^{\prime}\left(u_{1}(s, x)-u_{2}(s, x)\right)\left|Z_{1}(s, x)-Z_{2}(s, x)\right| d x d s \\
= & C \int_{t}^{T} \int_{D} \chi_{\left\{0 \leq u_{1}(s, x)-u_{2}(s, x) \leq \frac{1}{n}\right\}} n\left(u_{1}(s, x)-u_{2}(s, x)\right)^{2}\left|Z_{1}(s, x)-Z_{2}(s, x)\right| d x d s \\
& +C \int_{t}^{T} \int_{D} \chi_{\left\{u_{1}(s, x)-u_{2}(s, x)>\frac{1}{n}\right\}}\left[2\left(u_{1}(s, x)-u_{2}(s, x)\right)-\frac{1}{n}\right]\left|Z_{1}(s, x)-Z_{2}(s, x)\right| d x d s \\
\leq & C \int_{t}^{T} \int_{D} \chi_{\left\{u_{1}(s, x)-u_{2}(s, x)>\frac{1}{n}\right\}}\left(2\left(u_{1}(s, x)-u_{2}(s, x)\right)-\frac{1}{n}\right)^{2} d x d s \\
& +\int_{t}^{T} \int_{D} \chi_{\left\{u_{1}(s, x)-u_{2}(s, x)>\frac{1}{n}\right\}}\left|Z_{1}(s, x)-Z_{2}(s, x)\right|^{2} d x d s \\
& +\frac{1}{4} C^{2} \int_{t}^{T} \int_{D} \chi_{\left\{0 \leq u_{1}(s, x)-u_{2}(s, x) \leq \frac{1}{n}\right\}} n\left(u_{1}(s, x)-u_{2}(s, x)\right)^{3} d x d s \\
& +\int_{t}^{T} \int_{D} \chi_{\left\{0 \leq u_{1}(s, x)-u_{2}(s, x) \leq \frac{1}{n}\right\}} n\left(u_{1}(s, x)-u_{2}(s, x)\right)^{2}\left|Z_{1}(s, x)-Z_{2}(s, x)\right|^{2} d x d s \\
\leq & C^{\prime} \int_{t}^{T} \int_{D}\left(\left(u_{1}(s, x)-u_{2}(s, x)\right)^{+}\right)^{2} d x d s \\
& +\int_{t}^{T} \int_{D} \chi_{\left\{u_{1}(s, x)-u_{2}(s, x)>\frac{1}{n}\right\}}\left|Z_{1}(s, x)-Z_{2}(s, x)\right|^{2} d x d s \\
& +\int_{t}^{T} \int_{D} \chi_{\left\{0 \leq u_{1}(s, x)-u_{2}(s, x) \leq \frac{1}{n}\right\}} n\left(u_{1}(s, x)-u_{2}(s, x)\right)^{2}\left|Z_{1}(s, x)-Z_{2}(s, x)\right|^{2} d x d s
\end{aligned}
$$

(3.10),(3.11) and (3.12) imply that

$$
I_{n}^{3}+I_{n}^{5} \leq C \int_{t}^{T} \int_{D}\left(\left(u_{1}(s, x)-u_{2}(s, x)\right)^{+}\right)^{2} d x d s
$$

Thus it follows from (3.8), (3.9) and (3.13) that

$$
\begin{aligned}
& F_{n}\left(u_{1}(t)-u_{2}(t)\right) \\
\leq & F_{n}\left(\phi_{1}-\phi_{2}\right)+C \int_{t}^{T} \int_{D}\left(\left(u_{1}(s, x)-u_{2}(s, x)\right)^{+}\right)^{2} d x d s \\
& -\int_{t}^{T} F_{n}^{\prime}\left(u_{1}(s)-u_{2}(s)\right)\left(Z_{1}(s)-Z_{2}(s)\right) d B_{s}
\end{aligned}
$$

Take expectation and let $n \rightarrow \infty$ to get

$$
E\left[\int_{D}\left(\left(u_{1}(t, x)-u_{2}(t, x)\right)^{+}\right)^{2} d x\right] \leq \int_{t}^{T} d s E\left[\int_{D}\left(\left(u_{1}(s, x)-u_{2}(s, x)\right)^{+}\right)^{2} d x\right]
$$


Gronwall's inequality yields that

$$
E\left[\int_{D}\left(\left(u_{1}(t, x)-u_{2}(t, x)\right)^{+}\right)^{2} d x\right]=0
$$

which completes the proof of the theorem.

Remark. Comparison theorems for BSPDEs were also proved in [MYZ] and [HMY]. However, the results in these articles could not cover our theorem and the proofs are quite different.

We now proceed to prove existence and uniqueness of the reflected BSPDEs. Let $V=$ $W_{0}^{1,2}(D)$ be the Sobolev space of order one with the usual norm $\|\cdot\|$. As before let $K=L^{2}(D)$. Consider the reflected backward stochastic partial differential equation:

$$
\begin{aligned}
& d u(t)=-\frac{1}{2} \Delta u(t) d t-b(t, u(t, x), Z(t, x)) d t+Z(t, x) d B_{t}, t \in(0, T) \\
& \quad-\eta(d t, x), t \in(0, T), \\
& u(t, x) \geq L(t, x) \\
& \int_{0}^{T} \int_{D}(u(t, x)-L(t, x)) \eta(d t, x) d x=0, \\
& u(T, x)=\phi(x) \quad \text { a.s. }
\end{aligned}
$$

Theorem 3.2 Assume that $E\left[|\phi|_{K}^{2}\right]<\infty$ and that

$$
\left|b\left(s, u_{1}, z_{1}\right)-b\left(s, u_{2}, z_{2}\right)\right| \leq C\left(\left|u_{1}-u_{2}\right|+\left|z_{1}-z_{2}\right|\right) .
$$

Let $L(t, x)$ be a measurable function which is differentiable in $t$ and twice differentiable in $x$ such that

$$
\int_{0}^{T} \int_{D} L^{\prime}(t, x)^{2} d x d t<\infty, \int_{0}^{T} \int_{D}|\Delta L(t, x)|^{2} d x d t<\infty .
$$

Then there exists a unique $K \times L^{2}\left(D, \mathbb{R}^{m}\right) \times K$-valued progressively measurable process $(u(t, x), Z(t, x), \eta(t, x))$ such that

$$
E\left[\int_{0}^{T}\|u(t)\|_{V}^{2} d t\right]<\infty, \quad E\left[\int_{0}^{T}|Z(t)|_{L^{2}\left(D, R^{m}\right)}^{2} d t\right]<\infty .
$$

(ii) $\eta$ is a $K$-valued continuous process, non-negative and nondecreasing in $t$ and $\eta(0, x)=0$.

(iii) $u(t, x)=\phi(x)+\int_{t}^{T} \frac{1}{2} \Delta u(t, x) d s+\int_{t}^{T} b(s, u(s, x), Z(s, x)) d s-\int_{t}^{T} Z(s, x) d B_{s}$ $+\eta(T, x)-\eta(t, x) ; \quad 0 \leq t \leq T$, $u(t, x) \geq L(t, x) \quad$ a.e. $\quad x \in D, \forall t \in[0, T]$.

$$
\begin{gathered}
\int_{0}^{T} \int_{D}(u(t, x)-L(t, x)) \eta(d t, x) d x=0 \\
u(t, x)=u_{1}(t, x) ; \quad(t, x) \in(0, T) \times \partial D
\end{gathered}
$$


where $u(t)$ stands for the $K$-valued continuous process $u(t, \cdot)$ and (iii) is understood as an equation in the dual space $V^{*}$ of $V$.

For the proof of the theorem, we introduce the penalised BSPDEs:

$$
\begin{aligned}
d u^{n}(t)= & -\Delta u^{n}(t) d t-b\left(t, u^{n}(t, x), Z^{n}(t, x)\right) d t+Z^{n}(t, x) d B_{t} \\
& -n\left(u^{n}(t, x)-L(t, x)\right)^{-} d t, \quad t \in(0, T) \\
u^{n}(T, x)= & \phi(x) \quad \text { a.s. }
\end{aligned}
$$

According to $[\varnothing \mathrm{PZ}]$, the solution $\left(u^{n}, Z^{n}\right)$ of the above equation exists and is unique. We are going to show that the sequence $\left(u^{n}, Z^{n}\right)$ has a limit, which will be a solution of the equation (3.20). First we need some a priori estimates:

Lemma 3.3 Let $\left(u^{n}, Z^{n}\right)$ be the solution of equation (3.21). We have

$$
\begin{gathered}
\sup _{n} E\left[\sup _{t}\left|u^{n}(t)\right|_{K}^{2}\right]<\infty, \\
\sup _{n} E\left[\left.\int_{0}^{T}|| u^{n}(t)\right|_{V} ^{2}\right]<\infty, \\
\sup _{n} E\left[\int_{0}^{T}\left|Z^{n}(t)\right|_{L^{2}\left(D, R^{m}\right)}^{2}\right]<\infty .
\end{gathered}
$$

Proof. Take a function $f(t, x) \in C_{0}^{2,2}([-1, T+1] \times D)$ satisfying $f(t, x) \geq L(t, x)$. Applying Itô's formula, it follows that

$$
\begin{aligned}
\left|u^{n}(t)-f(t)\right|_{K}^{2}= & |\phi-f(T)|_{K}^{2}+2 \int_{t}^{T}<u^{n}(s)-f(s), \Delta u^{n}(s)>d s \\
& +2 \int_{t}^{T}<u^{n}(s)-f(s), b\left(s, u^{n}(s), Z^{n}(s)\right)>d s \\
& -2 \int_{t}^{T}<u^{n}(s)-f(s), Z^{n}(s)>d B_{s} \\
& +2 n \int_{t}^{T}<u^{n}(s)-f(s),\left(u^{n}(s)-L(s)\right)^{-}>d s-\int_{t}^{T}\left|Z^{n}(s)\right|_{L^{2}\left(D, \mathbb{R}^{m}\right)}^{2} d s \\
& +2 \int_{t}^{T}<u^{n}(s)-f(s), f^{\prime}(s)>d s, \quad \text { a.s. }
\end{aligned}
$$

where $<,>$ denotes the inner product in $K$. Now we estimate each of the terms on the right hand side: 


$$
\begin{gathered}
\quad 2 \int_{t}^{T}<u^{n}(s)-f(s), \Delta u^{n}(s)>d s \\
=-2 \int_{t}^{T}\left\|u^{n}(s)\right\|_{V}^{2} d s+2 \int_{t}^{T}<\frac{\partial f(s)}{\partial x}, \frac{\partial u^{n}(s)}{\partial x}>d s \\
\leq-\left.\int_{t}^{T}\left\|\left.u^{n}(s)\right|_{V} ^{2} d s+\int_{t}^{T}\right\| f(s)\right|_{V} ^{2} d s \\
\quad 2 \int_{t}^{T}<u^{n}(s)-f(s), b\left(s, u^{n}(s), Z^{n}(s)\right)>d s \\
=2 \int_{t}^{T}<u^{n}(s)-f(s), b\left(s, u^{n}(s), Z^{n}(s)\right)-b\left(s, f(s), Z^{n}(s)\right)>d s \\
+2 \int_{t}^{T}<u^{n}(s)-f(s), b\left(s, f(s), Z^{n}(s)\right)-b(s, f(s), 0)>d s \\
+2 \int_{t}^{T}<u^{n}(s)-f(s), b(s, f(s), 0)>d s \\
\leq \quad \int_{t}^{T}\left|u^{n}(s)-f(s)\right|_{H}^{2} d s+\frac{1}{2} \int_{t}^{T}\left|Z^{n}(s)\right|_{L^{2}\left(D, \mathbb{R}^{m}\right)}^{2} d s \\
+C \int_{t}^{T}|b(s, f(s), 0)|_{H}^{2} d s \\
2 n \int_{t}^{T}<\int_{D}^{T}\left(u^{n}(s)-f(s),\left(u^{n}(s)-L(s)\right)^{-}>d s\right. \\
=f(s, x)) \chi_{\left\{u^{n}(s, x) \leq L(s, x)\right\}}\left(L(s, x)-u^{n}(s, x)\right) d s d x \leq 0
\end{gathered}
$$

Substituting (3.27),(3.28) and (3.29) into (3.26) we obtain

$$
\begin{aligned}
& \left|u^{n}(t)-f(t)\right|_{K}^{2}+\int_{t}^{T}|| u^{n}(s) \|_{V}^{2} d s+\frac{1}{2} \int_{t}^{T}\left|Z^{n}(s)\right|_{L^{2}\left(D, \mathbb{R}^{m}\right)}^{2} d s \\
\leq & |\phi-f(T)|_{K}^{2}+C \int_{t}^{T}\left|u^{n}(s)-f(s)\right|_{K}^{2} d s+C \int_{t}^{T}|b(s, f(s), 0)|_{K}^{2} d s \\
& +\int_{t}^{T} \|\left. f(s)\right|_{V} ^{2} d s-2 \int_{t}^{T}<u^{n}(s)-f(s), Z^{n}(s)>d B_{s}
\end{aligned}
$$

We take expectation and use the Gronwall inequality to obtain

$$
\sup _{n} \sup _{t} E\left[\left|u^{n}(t)\right|_{K}^{2}\right]<\infty
$$




$$
\begin{gathered}
\sup _{n} E\left[\int_{0}^{T}\left\|u^{n}(t)\right\|_{V}^{2}\right]<\infty \\
\sup _{n} E\left[\int_{0}^{T}\left|Z^{n}(t)\right|_{L^{2}\left(D, \mathbb{R}^{m}\right)}^{2} d t\right]<\infty
\end{gathered}
$$

By virtue of (3.33), (3.31) can be further strengthend to (3.23). Indeed, by the Burkholder inequality,

$$
\begin{aligned}
& E\left[2 \sup _{v \leq t \leq T}\left|\int_{t}^{T}<u^{n}(s)-f(s), Z^{n}(s)>d B_{s}\right|\right] \\
\leq & C E\left[\left(\int_{v}^{T}\left|u^{n}(s)-f(s)\right|_{K}^{2}\left|Z^{n}(s)\right|_{L^{2}\left(D, \mathbb{R}^{m}\right)}^{2} d s\right)^{\frac{1}{2}}\right] \\
\leq & C E\left[\sup _{v \leq s \leq T}\left(\left|u^{n}(s)-f(s)\right|_{K}\right)\left(\int_{v}^{T}\left|Z^{n}(s)\right|_{L^{2}\left(D, \mathbb{R}^{m}\right)}^{2} d s\right)^{\frac{1}{2}}\right] \\
\leq & \frac{1}{2} E\left[\sup _{v \leq s \leq T}\left(\left|u^{n}(s)-f(s)\right|_{K}^{2}\right)\right]+C E\left[\int_{v}^{T}\left|Z^{n}(s)\right|_{L^{2}\left(D, \mathbb{R}^{m}\right)}^{2} d s\right]
\end{aligned}
$$

With (3.34), taking superum over $t \in[v, T]$ on both sides of (3.26) we obtain (3.23).

We need the following estimates:

Lemma 3.4 Suppose the conditions in Theorem 3.2 hold. Then there is a constant $C$ such that

$$
E\left[\int_{0}^{T} \int_{D}\left(\left(u^{n}(t, x)-L(t, x)\right)^{-}\right)^{2} d x d t\right] \leq \frac{C}{n^{2}} .
$$

Proof. Let $f_{m}$ be defined as in the proof of Theorem 3.1. Then $f_{m}(x) \uparrow\left(x^{+}\right)^{2}$ and $f_{m}^{\prime}(x) \uparrow$ $2 x^{+}$as $m \rightarrow \infty$. For $h \in K$, set

$$
G_{m}(h)=\int_{D} f_{m}(-h(x)) d x .
$$

It is easy to see that for $h_{1}, h_{2} \in K$,

$$
\begin{aligned}
G_{m}^{\prime}(h)\left(h_{1}\right) & =-\int_{D} f_{m}^{\prime}(-h(x)) h_{1}(x) d x, \\
G_{n}^{\prime \prime}(h)\left(h_{1}, h_{2}\right) & =\int_{D} f_{m}^{\prime \prime}(-h(x)) h_{1}(x) h_{2}(x) d x .
\end{aligned}
$$


Applying Itô's formula we get

$$
\begin{aligned}
& G_{m}\left(u^{n}(t)-L(t)\right) \\
= & \left.G_{m}(\phi-L(T))+\int_{t}^{T} G_{m}^{\prime}\left(u^{n}(s)-L(s)\right)\left(\Delta u^{n}(s)\right)\right) d s \\
& +\int_{t}^{T} G_{m}^{\prime}\left(u^{n}(s)-L(s)\right)\left(b\left(s, u^{n}(s), Z^{n}(s)\right)\right) d s \\
& +n \int_{t}^{T} G_{m}^{\prime}\left(u^{n}(s)-L(s)\right)\left(\left(u^{n}(s)-L(s)\right)^{-}\right) d s \\
& +\int_{t}^{T} G_{m}^{\prime}\left(u^{n}(s)-L(s)\right)\left(L^{\prime}(s)\right) d s \\
& -\int_{t}^{T} G_{m}^{\prime}\left(u^{n}(s)-L(s)\right)\left(Z^{n}(s)\right) d B_{s} \\
& -\frac{1}{2} \int_{t}^{T} G_{m}^{\prime \prime}\left(Z^{n}(s), Z^{n}(s)\right) d s \\
=: & I_{m}^{1}+I_{m}^{2}+I_{m}^{3}+I_{m}^{4}+I_{m}^{5}+I_{m}^{6}+I_{m}^{7} .
\end{aligned}
$$

Now,

$$
\begin{aligned}
& \left.I_{m}^{2}=\int_{t}^{T} G_{m}^{\prime}\left(u^{n}(s)-L(s)\right)\left(\Delta u^{n}(s)\right)\right) d s \\
= & -\int_{t}^{T} \int_{D} f_{m}^{\prime}\left(L(s, x)-u^{n}(s, x)\right)\left(\Delta\left(u^{n}(s, x)-L(s, x)\right)\right) d x d s \\
& -\int_{t}^{T} \int_{D} f_{m}^{\prime}\left(L(s, x)-u^{n}(s, x)\right)(\Delta L(s, x)) d x d s \\
\leq & -\int_{t}^{T} \int_{D} f_{m}^{\prime \prime}\left(L(s, x)-u^{n}(s, x)\right)\left|\nabla\left(u^{n}(s, x)-L(s, x)\right)\right|^{2} d x d s \\
& +\frac{1}{4} n \int_{t}^{T} \int_{D} f_{m}^{\prime}\left(L(s, x)-u^{n}(s, x)\right)^{2} x d s \\
& +\frac{C}{n} \int_{t}^{T} \int_{D}(\Delta L(s, x))^{2} d x d s, \\
I_{m}^{3}= & -\int_{t}^{T} \int_{D} f_{m}^{\prime}\left(L(s, x)-u^{n}(s, x)\right) b\left(s, u^{n}(s, x), Z^{n}(s, x)\right) d x d s \\
\leq & \frac{1}{4} n \int_{t}^{T} \int_{D} f_{m}^{\prime}\left(L(s, x)-u^{n}(s, x)\right)^{2} d s \\
+ & \frac{C}{n} \int_{t}^{T} \int_{D}\left(b\left(s, u^{n}(s, x), Z^{n}(s, x)\right)\right)^{2} d x d s,
\end{aligned}
$$




$$
\begin{aligned}
I_{m}^{5} & =-\int_{t}^{T} \int_{D} f_{m}^{\prime}\left(L(s, x)-u^{n}(s, x)\right)\left(L^{\prime}(s, x)\right) d x d s \\
& \leq \frac{1}{4} n \int_{t}^{T} \int_{D} f_{m}^{\prime}\left(L(s, x)-u^{n}(s, x)\right)^{2} d s \\
& +\frac{C}{n} \int_{t}^{T} \int_{D}\left(L^{\prime}(s, x)\right)^{2} d x d s .
\end{aligned}
$$

Combining (3.38)-(3.41) and taking expectation we obtain

$$
\begin{aligned}
& E\left[G_{m}\left(u^{n}(t)-L(t)\right)\right] \\
\leq & E\left[G_{m}(\phi-L(T))\right]+\frac{3}{4} n \int_{t}^{T} \int_{D} f_{m}^{\prime}\left(L(s, x)-u^{n}(s, x)\right)^{2} d s \\
+ & \frac{C}{n} E\left[\int_{t}^{T} \int_{D}\left(L^{\prime}(s, x)\right)^{2} d x d s\right]+\frac{C}{n} E\left[\int_{t}^{T} \int_{D}(\Delta L(s, x))^{2} d x d s\right] \\
+ & \frac{C}{n} E\left[\int_{t}^{T} \int_{D}\left(b\left(s, u^{n}(s, x), Z^{n}(s, x)\right)\right)^{2} d x d s\right] \\
- & n E\left[\int_{t}^{T} \int_{D} f_{m}^{\prime}\left(L(s, x)-u^{n}(s, x)\right)\left(\left(u^{n}(s, x)-L(s, x)\right)^{-}\right) d s\right] .
\end{aligned}
$$

Letting $m \rightarrow \infty$ we conclude that

$$
\begin{aligned}
& E\left[\int_{D}\left(\left(u^{n}(t, x)-L(t, x)\right)^{-}\right)^{2} d x\right] \\
\leq & \frac{3}{4} n E\left[\int_{t}^{T} \int_{D}\left(\left(u^{n}(s, x)-L(s, x)\right)^{-}\right)^{2} d x d s\right] \\
& -n E\left[\int_{t}^{T} \int_{D}\left(\left(u^{n}(s, x)-L(s, x)\right)^{-}\right)^{2} d x d s\right]+\frac{C^{\prime}}{n},
\end{aligned}
$$

where the Lipschiz condition of $b$ and Lemma 3.3 have been used. In particular we have

$$
E\left[\int_{t}^{T} \int_{D}\left(\left(u^{n}(s, x)-L(s, x)\right)^{-}\right)^{2} d x d s\right] \leq \frac{C^{\prime}}{n^{2}} .
$$

Lemma 3.5 Let $\left(u^{n}, Z^{n}\right)$ be the solution of equation (3.21). We have

$$
\lim _{n, m \rightarrow \infty} E\left[\sup _{0 \leq t \leq T}\left|u^{n}(t)-u^{m}(t)\right|_{K}^{2}\right]=0,
$$




$$
\begin{gathered}
\lim _{n, m \rightarrow \infty} E\left[\int_{0}^{T}\left\|u^{n}(t)-u^{m}(t)\right\|_{V}^{2} d t\right]=0 . \\
\lim _{n, m \rightarrow \infty} E\left[\int_{0}^{T}\left|Z^{n}(t)-Z^{m}(t)\right|_{L^{2}\left(D, \mathbb{R}^{m}\right)}^{2} d t\right]=0 .
\end{gathered}
$$

Proof. Applying Itô's formula, it follows that

$$
\begin{aligned}
& \left|u^{n}(t)-u^{m}(t)\right|_{K}^{2} \\
= & 2 \int_{t}^{T}<u^{n}(s)-u^{m}(s), \Delta\left(u^{n}(s)-u^{m}(s)\right)>d s \\
& +2 \int_{t}^{T}<u^{n}(s)-u^{m}(s), b\left(s, u^{n}(s), Z^{n}(s)\right)-b\left(s, u^{m}(s), Z^{m}(s)\right)>d s \\
& -2 \int_{t}^{T}<u^{n}(s)-u^{m}(s), Z^{n}(s)-Z^{m}(s)>d B_{s} \\
& +2 \int_{t}^{T}<u^{n}(s)-u^{m}(s), n\left(u^{n}(s)-L(s)\right)^{-}-m\left(u^{m}(s)-L(s)\right)^{-}>d s \\
& -\int_{t}^{T}\left|Z^{n}(s)-Z^{m}(s)\right|_{L^{2}\left(D, \mathbb{R}^{m}\right)}^{2} d s
\end{aligned}
$$

Now we estimate each of the terms on the right side:

$$
\begin{aligned}
& 2 \int_{t}^{T}<u^{n}(s)-u^{m}(s), \Delta\left(u^{n}(s)-u^{m}(s)\right)>d s \\
= & -2 \int_{t}^{T}\left\|u^{n}(s)-u^{m}(s)\right\|_{V}^{2} d s .
\end{aligned}
$$

By the Lipschitz continuity of $b$ and the inequality $a b \leq \varepsilon a^{2}+C_{\varepsilon} b^{2}$, one has

$$
\begin{aligned}
& 2 \int_{t}^{T}<u^{n}(s)-u^{m}(s), b\left(s, u^{n}(s), Z^{n}(s)\right)-b\left(s, u^{m}(s), Z^{m}(s)\right)>d s \\
\leq & C \int_{t}^{T}\left|u^{n}(s)-u^{m}(s)\right|_{K}^{2} d s+\frac{1}{2} \int_{t}^{T}\left|Z^{n}(s)-Z^{m}(s)\right|_{L^{2}\left(D, \mathbb{R}^{m}\right)}^{2} d s .
\end{aligned}
$$


In view of (3.44),

$$
\begin{aligned}
& 2 E\left[\int_{t}^{T}<u^{n}(s)-u^{m}(s), n\left(u^{n}(s)-L(s)\right)^{-}-m\left(u^{m}(s)-L(s)\right)^{-}>d s\right] \\
= & 2 n E\left[\int_{t}^{T}<u^{n}(s)-L(s),\left(u^{n}(s)-L(s)\right)^{-}>d s\right] \\
& +2 m E\left[\int_{t}^{T}<L(s)-u^{n}(s),\left(u^{m}(s)-L(s)\right)^{-}>d s\right] \\
& +2 m E\left[\int_{t}^{T}<u^{m}(s)-L(s),\left(u^{m}(s)-L(s)\right)^{-}>d s\right] \\
& +2 n E\left[\int_{t}^{T}<L(s)-u^{m}(s),\left(u^{n}(s)-L(s)\right)^{-}>d s\right] \\
\leq & 2 m E\left[\int_{t}^{T}<L(s)-u^{n}(s),\left(u^{m}(s)-L(s)\right)^{-}>d s\right] \\
& +2 n E\left[\int_{t}^{T}<L(s)-u^{m}(s),\left(u^{n}(s)-L(s)\right)^{-}>d s\right] \\
\leq & 2 m E\left[\int_{t}^{T} \int_{D}\left(u^{n}(s, x)-L(s, x)\right)^{-}\left(u^{m}(s, x)-L(s, x)\right)^{-} d x d s\right] \\
& +2 n E\left[\int_{t}^{T} \int_{D}\left(u^{m}(s, x)-L(s, x)\right)^{-}\left(u^{n}(s, x)-L(s, x)\right)^{-} d x d s\right] \\
\leq & 2 m\left(E\left[\int_{t}^{T} \int_{D}\left(\left(u^{n}(s, x)-L(s, x)\right)^{-}\right)^{2} d x d s\right]\right)^{\frac{1}{2}}\left(E\left[\int_{t}^{T} \int_{D}\left(\left(u^{m}(s, x)-L(s, x)\right)^{-}\right)^{2} d x d s\right]\right)^{\frac{1}{2}} \\
& +2 n\left(E\left[\int_{t}^{T} \int_{D}\left(\left(u^{n}(s, x)-L(s, x)\right)^{-}\right)^{2} d x d s\right]\right)^{\frac{1}{2}}\left(E\left[\int_{t}^{T} \int_{D}\left(\left(u^{m}(s, x)-L(s, x)\right)^{-}\right)^{2} d x d s\right]\right)^{\frac{1}{2}} \\
& +3.51)
\end{aligned}
$$

It follows from (3.48) and (3.49) that

$$
\begin{aligned}
& E\left[\left|u^{n}(t)-u^{m}(t)\right|_{K}^{2}\right]+\frac{1}{2} E\left[\int_{t}^{T}\left|Z^{n}(s)-Z^{m}(s)\right|_{L^{2}\left(D, \mathbb{R}^{m}\right)}^{2} d s\right] \\
& +E\left[\int_{t}^{T} \| u^{n}(s)-\left.u^{m}(s)\right|_{V} ^{2} d s\right] \\
\leq & C \int_{t}^{T} E\left[\left|u^{n}(s)-u^{m}(s)\right|_{K}^{2}\right] d s+C^{\prime}\left(\frac{1}{n}+\frac{1}{m}\right) .
\end{aligned}
$$

Application of the Gronwall inequality yields

$$
\lim _{n, m \rightarrow \infty}\left\{E\left[\left|u^{n}(t)-u^{m}(t)\right|_{K}^{2}\right]+\frac{1}{2} E\left[\int_{t}^{T}\left|Z^{n}(s)-Z^{m}(s)\right|_{L^{2}\left(D, \mathbb{R}^{m}\right)}^{2} d s\right]\right\}=0,
$$




$$
\lim _{n, m \rightarrow \infty} E\left[\int_{t}^{T}\left\|u^{n}(s)-u^{m}(s)\right\|_{V}^{2} d s\right]=0 .
$$

By (3.53) and the Burkholder inequality we can further show that

$$
\lim _{n, m \rightarrow \infty} E\left[\sup _{0 \leq t \leq T}\left|u^{n}(t)-u^{m}(t)\right|_{K}^{2}\right]=0 .
$$

The proof is complete.

Proof of Theorem 3.2. From Lemma 3.5 we know that $\left(u^{n}, Z^{n}\right), n \geq 1$, forms a Cauchy sequence. Denote by $u(t, x), Z(t, x)$ the limit of $u^{n}$ and $Z^{n}$. Put

$$
\bar{\eta}^{n}(t, x)=n\left(u^{n}(t, x)-L(t, x)\right)^{-}
$$

Lemma 3.4 implies that $\bar{\eta}^{n}(t, x)$ admits a non-negative weak limit, denoted by $\bar{\eta}(t, x)$, in the following Hilbert space:

$$
\bar{K}=\left\{h ; \quad \mathrm{h} \text { is a K-valued adapted process such that } E\left[\int_{0}^{T}|h(s)|_{K}^{2} d s\right]<\infty\right\}
$$

with inner product

$$
<h_{1}, h_{2}>_{\bar{K}}=E\left[\int_{0}^{T} \int_{D} h_{1}(t, x) h_{2}(t, x) d t d x\right] .
$$

Set $\eta(t, x)=\int_{0}^{t} \bar{\eta}(s, x) d s$. Then $\eta$ is a continuous $K$-valued process which is increasing in $t$. Keeping Lemma 3.5 in mind and letting $n \rightarrow \infty$ in (3.21) we obtain

$$
\begin{aligned}
& u(t, x) \\
= & \phi(x)+\int_{t}^{T} \Delta u(t, x) d s+\int_{t}^{T} b(s, u(s, x), Z(s, x)) d s-\int_{t}^{T} Z(s, x) d B_{s} \\
& +\eta(T, x)-\eta(t, x) ; \quad 0 \leq t \leq T .
\end{aligned}
$$

Recall from Lemma 3.4 that

$$
E\left[\int_{t}^{T} \int_{D}\left(\left(u^{n}(s, x)-L(s, x)\right)^{-}\right)^{2} d x d s\right] \leq C^{\prime} \frac{1}{n^{2}}
$$

By the Fatou Lemma, this implies that $E\left[\int_{t}^{T} \int_{D}\left((u(s, x)-L(s, x))^{-}\right)^{2} d x d s\right]=0$. In view of the continuity of $u$ in $t$, we conclude $u(t, x) \geq L(t, x)$ a.e. in $x$, for every $t \geq 0$. Combining the strong convergence of $u^{n}$ and the weak convergence of $\bar{\eta}^{n}$, we also have

$$
\begin{aligned}
& E\left[\int_{0}^{T} \int_{D}(u(s, x)-L(s, x)) \eta(d t, x) d x\right] \\
= & E\left[\int_{0}^{T} \int_{D}(u(s, x)-L(s, x)) \bar{\eta}(t, x) d t d x\right] \\
\leq & \lim _{n \rightarrow \infty} E\left[\int_{0}^{T} \int_{D}\left(u^{n}(s, x)-L(s, x)\right) \bar{\eta}^{n}(t, x) d t d x\right] \leq 0
\end{aligned}
$$


Hence,

$$
\int_{0}^{T} \int_{D}(u(s, x)-L(s, x)) \eta(d t, x) d x=0, \quad \text { a.s. }
$$

We have shown that $(u, Z, \eta)$ is a solution to the reflected BSPDE (3.17).

Uniqueness. Let $\left(u_{1}, Z_{1}, \eta_{1}\right),\left(u_{2}, Z_{2}, \eta_{2}\right)$ be two such solutions to equation (3.20). By Itô's formula, we have

$$
\begin{aligned}
& \left|u_{1}(t)-u_{2}(t)\right|_{K}^{2} \\
= & 2 \int_{t}^{T}<u_{1}(s)-u_{2}(s), \Delta\left(u_{1}(s)-u_{2}(s)\right)>d s \\
& +2 \int_{t}^{T}<u_{1}(s)-u_{2}(s), b\left(s, u_{1}(s), Z_{1}(s)\right)-b\left(s, u_{2}(s), Z_{2}(s)\right)>d s \\
& -2 \int_{t}^{T}<u_{1}(s)-u_{2}(s), Z_{1}(s)-Z_{2}(s)>d B_{s} \\
& +2 \int_{t}^{T}<u_{1}(s)-u_{2}(s), \eta_{1}(d s)-\eta_{2}(d s)> \\
& -\int_{t}^{T}\left|Z_{1}(s)-Z_{2}(s)\right|_{L^{2}\left(D, \mathbb{R}^{m}\right)}^{2} d s
\end{aligned}
$$

Similar to the proof of Lemma 3.5, we have

$$
2 \int_{t}^{T}<u_{1}(s)-u_{2}(s), \Delta\left(u_{1}(s)-u_{2}(s)\right)>d s \leq 0,
$$

and

$$
\begin{aligned}
& 2 \int_{t}^{T}<u_{1}(s)-u_{2}(s), b\left(s, u_{1}(s), Z_{1}(s)\right)-b\left(s, u_{2}(s), Z_{2}(s)\right)>d s \\
\leq & C \int_{t}^{T}\left|u_{1}(s)-u_{2}(s)\right|_{K}^{2} d s+\frac{1}{2} \int_{t}^{T}\left|Z_{1}(s)-Z_{2}(s)\right|_{L^{2}\left(D, \mathbb{R}^{m}\right)}^{2} d s
\end{aligned}
$$


On the other hand,

$$
\begin{aligned}
& 2 E\left[\int_{t}^{T}<u_{1}(s)-u_{2}(s), \eta_{1}(d s)-\eta_{2}(d s)>\right] \\
= & 2 E\left[\int_{t}^{T} \int_{D}\left(u_{1}(s, x)-L(s, x)\right) \eta_{1}(d s, x) d x\right] \\
& -2 E\left[\int_{t}^{T} \int_{D}\left(u_{1}(s, x)-L(s, x)\right) \eta_{2}(d s, x) d x\right] \\
& +2 E\left[\int_{t}^{T} \int_{D}\left(u_{2}(s, x)-L(s, x)\right) \eta_{2}(d s, x) d x\right] \\
& -2 E\left[\int_{t}^{T} \int_{D}\left(u_{2}(s, x)-L(s, x)\right) \eta_{1}(d s, x) d x\right] \\
\leq & 0
\end{aligned}
$$

Combining (3.58) - (3.61) we arrive at

$$
\begin{aligned}
& E\left[\left|u_{1}(t)-u_{2}(t)\right|_{K}^{2}\right]+\frac{1}{2} E\left[\int_{t}^{T}\left|Z_{1}(s)-Z_{2}(s)\right|_{L^{2}\left(D, \mathbb{R}^{m}\right)}^{2} d s\right] \\
\leq & C \int_{t}^{T} E\left[\left|u_{1}(s)-u_{2}(s)\right|_{K}^{2}\right] d s .
\end{aligned}
$$

Appealing to the Gronwall inequality, this implies

$$
u_{1}=u_{2}, \quad Z_{1}=Z_{2}
$$

which further gives $\eta_{1}=\eta_{2}$ from the equation they satisfy.

\section{$4 \quad$ Link to optimal stopping}

In this section, we provide a link between the solution of a reflected backward stochastic partial differential equation and an optimal stopping problem.

Let $u(t, x), Z(t, x), \eta(t, x)$ be the solution of the following reflected BSPDE.

$$
\begin{aligned}
& u(t, x) \\
= & \phi(x)+\int_{t}^{T} \frac{1}{2} \Delta u(s, x) d s+\int_{t}^{T} g(s, x, u(s, x), Z(s, x)) d s-\int_{t}^{T} Z(s, x) d B_{s} \\
& +\eta(T, x)-\eta(t, x) ; \quad 0 \leq t \leq T, \\
& u(t, x) \geq L(t, x), \quad(t, x) \in[0, T] \times \mathbb{R}^{d}, \\
& \int_{0}^{T} \int_{D}(u(s, x)-L(s, x)) \eta(d s, x) d x=0 \quad \text { a.s. }
\end{aligned}
$$


Choose an adapted process $\hat{Z}(t, x)$. Let $\mathcal{S}_{t, T}$ be the set of all stopping times $\tau$ satisfying $t \leq \tau \leq T$. For $\tau \in \mathcal{S}_{t, T}$, define

$$
Y^{\tau}(t, x)=\int_{t}^{\tau} P_{s-t} g(s, x) d s+P_{\tau-t} L(\tau, x) \chi_{\{\tau<T\}}+P_{\tau-t} \phi(x) \chi_{\{\tau=T\}}-\int_{t}^{\tau} P_{s-t} \hat{Z}(s, x) d B_{s},
$$

where $g(s, \cdot)=g(s, \cdot, u(s, \cdot), Z(s, \cdot))$ and $P_{t}$ denotes the semigroup generated by the Laplacian operator $\frac{1}{2} \Delta$, i.e.

$$
P_{t} f(x)=(2 \pi t)^{-d / 2} \int_{\mathbb{R}^{d}} f(y) \exp \left(-\frac{|y-x|^{2}}{2 t}\right) d y ; f \in L^{1}\left(\mathbb{R}^{d}\right) .
$$

Here, and in the following we will use the simplified notation $P_{t} g(s, x)=\left(P_{t} g(s, \cdot)\right)(x)$ etc.

Theorem $4.1 u(t, x)$ is the value function of the the optimal stopping problem associated with $Y^{\tau}(t, x)$, i.e.,

$$
u(t, x)=\operatorname{essup}_{\tau \in \mathcal{S}_{t, T}} E\left[Y^{\tau}(t, x) \mid \mathcal{F}_{t}\right]
$$

Moreover,

$$
\hat{\tau}:=\hat{\tau}(t, x):=\inf \{s \in[t, T) \mid u(s, x)=L(s, x)\} \wedge T
$$

is an optimal stopping time.

Proof. Observe that $u$ admits the following mild representation:

$$
\begin{aligned}
& u(t, x)=P_{T-t} \phi(x)+\int_{t}^{T} P_{s-t}(g(s, u(s, x), Z(s, x))) d s-\int_{t}^{T} P_{s-t}(Z(s, x)) d B_{s} \\
& +\int_{t}^{T} P_{s-t} \eta(d s, x) ; \quad 0 \leq t \leq T .
\end{aligned}
$$

More generally, for any stopping time $\tau$ with $t \leq \tau \leq T$, we have

$$
\begin{aligned}
& u(t, x)=P_{\tau-t}(u(\tau, x))+\int_{t}^{\tau} P_{s-t}(g(s, x)) d s-\int_{t}^{\tau} P_{s-t}(Z(s, x)) d B(s) \\
& +\int_{t}^{\tau} P_{s-t} \eta(d s, x) ; \quad 0 \leq t \leq \tau .
\end{aligned}
$$

Since $\eta(s, x)$ is increasing in $s$ and $u(s, x) \geq L(s, x)$ for $s \leq T$, it follows that

$$
u(t, x) \geq Y^{\tau}(t, x)-\int_{t}^{\tau} P_{s-t}(Z(s, x)) d B(s)+\int_{t}^{\tau} P_{s-t}(\hat{Z}(s, x)) d B_{s}
$$

Taking conditional expectation with respect to $\mathcal{F}_{t}$ on both sides we get

$$
u(t, x) \geq E\left[Y^{\tau}(t, x) \mid \mathcal{F}_{t}\right]
$$


As $\tau$ is arbitrary, we obtain

$$
u(t, x) \geq e s s \sup _{\tau \in \mathcal{S}_{t, T}} E\left[Y^{\tau}(t, x) \mid \mathcal{F}_{t}\right]
$$

Now, define

$$
\hat{\tau}=\hat{\tau}(t, x)=\inf \{s \in[t, T) \mid u(s, x)=L(s, x)\} \wedge T .
$$

From the property of $\eta$, it is not increasing on the interval $[t, \hat{\tau}]$. Thus, $\int_{t}^{\hat{\tau}} P_{s-t} \eta(d s, x)=0$. So we have from (4.6) that

$$
\begin{aligned}
& u(t, x)=\left.P_{\tau-t}(u(\tau, x))\right|_{\tau=\hat{\tau}}+\int_{t}^{\hat{\tau}} P_{s-t}(g(s, x, u(s, x), Z(s, x))) d s \\
& -\int_{t}^{\hat{\tau}} P_{s-t}(Z(s, x)) d B_{s}+\int_{t}^{\hat{\tau}} P_{s-t}(\hat{Z}(s, x)) d B_{s} \\
& =\left.Y^{\tau}(t, x)\right|_{\tau=\hat{\tau}}-\int_{t}^{\hat{\tau}} P_{s-t}(Z(s, x)) d B_{s}+\int_{t}^{\hat{\tau}} P_{s-t}(\hat{Z}(s, x)) d B_{s} \\
& =Y^{\hat{\tau}}(t, x)-\int_{t}^{\hat{\tau}} P_{s-t}(Z(s, x)) d B_{s}+\int_{t}^{\hat{\tau}} P_{s-t}(\hat{Z}(s, x)) d B_{s} .
\end{aligned}
$$

Taking conditional expectation yields that

$$
u(t, x)=E\left[Y^{\hat{\tau}} \mid \mathcal{F}_{t}\right]
$$

Combining this with (4.7) we obtain the theorem.

\section{Application to risk minimising stopping}

Let $\tau \in \mathcal{S}_{0, T}$, the set of stopping times with values between 0 and $T$. Suppose that $g(s, x, Z)$ is convex with respect to $Z$ for all $s, x$. Let $F(t, x)$ be a given square integrable adapted process. In analogy with the definition of a convex risk measure in finance in terms of (ordinary) backward stochastic differential equations, we may consider $F^{\tau}(x)=F(\tau, x)$ as the financial standing at $(\tau, x)$, and we define the risk $\rho\left(F^{\tau}\right)(t, x)$ of $F^{\tau}(x)$ at time $t \leq \tau$ and at the point $x$ by

$$
\rho\left(F^{\tau}\right)(t, x)=-Y_{F^{\tau}}(t, x),
$$

where $Y(t, x)=Y_{F^{\tau}}(t, x), \hat{Z}(t, x)$ is the solution of the BSPDE

$$
\begin{aligned}
& d Y(t, x)=-\frac{1}{2} \Delta Y(t, x) d t-g(t, x, Z(t, x)) d t+\hat{Z}(t, x) d B(t),(t, x) \in(0, \tau) \times \mathbb{R}^{d} \\
& Y(\tau, x)=F^{\tau}(x) ; x \in \mathbb{R}^{d}
\end{aligned}
$$


We consider the risk minimising stopping problem to find $\tau^{*} \in \mathcal{S}_{0, T}$ and $\rho\left(F^{\tau^{*}}\right)(t, x)$ such that

$$
\rho\left(F^{\tau^{*}}\right)(t, x)=i n f_{\tau \in \mathcal{S}_{t, T}} \rho\left(F^{\tau}\right)(t, x)
$$

We may consider the space diffusion effect stemming from the Laplacian operator, as a representation of a mean-field effect in a market with many agents with interacting notions of risk.

Note that the solution of the BSPDE for $Y_{F^{\tau}}(t, x)$ is

$$
Y_{F^{\tau}}(t, x)=\int_{t}^{\tau} P_{s-t} g(s, x) d s+P_{\tau-t} F(\tau, x)-\int_{t}^{\tau} P_{s-t}(\hat{Z}(s, x)) d B(s) .
$$

Therefore, comparing with the equation (4.6) above for $Y^{\tau}(t, x)$, we see that $Y_{F}^{\tau}(t, x)$ coincides with $Y^{\tau}(t, x)$ if we choose $L(t, x)$ and $\phi(x)$ such that

$$
F(t, x)=L(t, x) \chi_{t<T}+\phi(x) \chi_{t=T}
$$

Applying the Theorem above to this choice of $L(t, x)$ and $\phi(x)$ we get the following result, which is a space-time version of a known result (see Quenez-Sulem (2012)):

Theorem 5.1 (Risk minimising stopping theorem)

$$
i n f_{\tau \in \mathcal{S}_{t, T}} \rho\left(F^{\tau}\right)(t, x)=-u(t, x),
$$

where $u(t, x), Z(t, x), \eta(t, x)$ is the solution of the reflected BSPDE

$$
\begin{aligned}
& u(t, x)=F(T, x)+\int_{t}^{T} \frac{1}{2} \Delta u(s, x) d s+\int_{t}^{T} g(s, x, u(s, x), Z(s, x)) d s \\
& -\int_{t}^{T} Z(s, x) d B(s)+\eta(T, x)-\eta(t, x) ; \quad(t, x) \in(0, T) \times \mathbb{R}^{d}, \\
& u(t, x) \geq F(t, x) ; \quad(t, x) \in(0, T) \times \mathbb{R}^{d}, \\
& \int_{0}^{T} \int_{\mathbb{R}^{d}}(u(s, x)-F(s, x)) \eta(d s, x) d x=0 \quad \text { a.s. }
\end{aligned}
$$

Moreover, the stopping time $\hat{\tau}=\hat{\tau}(t, x)$ defined by

$$
\hat{\tau}(t, x)=\inf \{s \in[t, T) \mid u(s, x)=F(s, x)\} \wedge T
$$

is optimal. 


\section{References}

[B] A. Bensoussan, Stochastic maximum principle for distributed parameter systems. J. Franklin Inst. 315 (1983), no. 5-6, 387-406.

[AND] D. Andersson: The relaxed general maximum principle for singular optimal control of diffusions. Systems $\&$ Control Letters 58 (2009), 76-82.

[BM] S. Bahlali and B. Mezerdi: A general stochastic maximum principle for singular control problems. Electronic J. Probab. 10 (2005), 988-1004.

[BDM] S. Bahlali, B. Djehiche and B. Mezerdi: The relaxed stochastic maximum principles in singular optimal control of diffusions. SIAM J. Cont. and Opt., 46(2) (2007), 427-444.

[BCDM] S. Bahlali, F. Chighoub, B. Djehiche and B. Mezerdi: Optimality necessary conditions in singular stochastic control problems with non-smooth coefficients. J. Math. Anal. Appl. 355 (2009), 479-494.

[DP1] C. Donati-Martin, E. Pardoux: White noise driven SPDEs with reflection. Probab. Theory Rel. Fields 95 (1993), 1-24.

[GP] A. Gegout-Petit, E. Pardoux: Equations Différentielles Stochastiques Rétrogrades Réfléchies Dans Un Convexe. Stochastics and Stochastics Reports 57 (1996), 111-128.

[GM] G. Guatteri and F. Masiero: On the existence of optimal controls for SPDEs with boundary-noise and boundary-control, arXiv:1110.6534v1

[HP] U. G. Haussmann, E. Pardoux: Stochastic variational inequalities of parabolic type. Appl. Math. Optim. 20(1989), 163-192.

[HP1] Hu and Peng, Adapted solution of a backward semilinear stochastic evolu- tion equation. Stochastic Anal. Appl. 9 (1991), no. 4, 445-459.

[HP2] Hu and Peng, Maximum principle for semilinear stochastic evolution control systems. Stochastics Stochastics Rep. 33 (1990), no. 3-4, 159-180.

[HMY] Hu, Ma and Yong, On semi-linear degenerate backward stochastic partial differential equations. Probab. Theory Related Fields 123 (2002), no. 3, 381-411.

[MYZ] J. Ma, H. Yin and J. Zhang: On non-Markovian forward-backward SDEs and backward stochastic PDEs. Manuscript June 16, 2011 (USC, Los Angeles).

[NP] D. Nualart, E. Pardoux: White noise driven by quasilinear SPDEs with reflection. Probab. Theory Rel. Fields 93 (1992), 77-89.

[ØS] B. Øksendal, A. Sulem: Singular stochastic control and optimal stopping with partial information of jump diffusions. SIAM J. Control and Optimization (to appear). 
[ØPZ] B. Øksendal, F. Proske and T. Zhang: Backward stochastic partial differential equations with jumps and application to optimal control of random jump fields. Stochastics 77:5 (2005), 381-399.

[P] E.Pardoux: Stochastic partial differential equations and filtering of diffussion processes. Stochastics 3 (1979), 127-167.

[PP1] E. Pardoux and S. Peng: Adapted solutions of backward stochastic differential equations. Systems and Control Letters 14 (1990), 55-61.

[PP2] E. Pardoux and S. Peng: Backward doubly stochastic differential equations and systems of quasilinear stochastic partial differential equations. Probab. Theory and Rel. Fields 98 (1994), 209-227.

[PR] C. Prévôt and M. Röckner: A concise Course on Stochastic Partial Differential Equations. Lecture Notes in Mathematics 1905, Springer 2007.

[QS] M.-C. Quenez and A. Sulem: Reflected BSDEs with jumps and application to optimal stopping for dynamic risk measures. Manuscript 2011.

[Z] T.Zhang : White noise driven SPDEs with reflection: strong Feller properties and Harnack inequalities. Potential Analysis 33:2 (2010), 137-151. 